\title{
Membrane scaffolds enhance the responsiveness and stability of DNA-based sensing circuits
}

\author{
Will T. Kaufhold, Ryan A. Brady, Joshua M. Tuffnell, Pietro Cicuta, and \\ Lorenzo Di Michele* \\ Biological and Soft Systems, Cavendish Laboratory, University of Cambridge, JJ Thomson \\ Av, Cambridge CB3 OHE, United Kingdom \\ E-mail: Id389@cam.ac.uk
}

\begin{abstract}
Target-induced DNA strand displacement is an excellent candidate for developing analyteresponsive DNA circuitry to be used in clinical diagnostics and synthetic biology. While most available technologies rely on DNA circuitry free to diffuse in bulk, here we explore the use of liposomes as scaffolds for DNA-based sensing nanodevices. Our proof-of-concept sensing circuit responds to the presence of a model target analyte by releasing a DNA strand, which in turn activates a fluorescent reporter. Through a combination of experiments and coarse-grained Monte Carlo simulations, we demonstrate that the presence of the membrane scaffold accelerates the process of oligonucleotide release and suppresses undesired leakage reactions, making the sensor both more responsive and robust.
\end{abstract}

\section{Introduction}

DNA has been abstracted from its cellular role in the storage of genetic information, and its base pairing interactions have been exploited to create a diverse range of self-assembling nanodevices and materials $(1-5)$. The programmability of DNA association can be employed also to design chemical reaction networks, whose most ubiquitous mechanism is toehold-mediated strand displacement $(6,7)$. In this reaction, hybridization of an invader single-stranded (ss) DNA molecule to a short toehold region on a substrate strand allows the invader to displace and release an incumbent strand initially bound to the substrate, after a period of reversible branch migration $(6,7)$.

Target-induced DNA strand displacement (TIDSD) can be seen as a conceptual extension of the toeholding mechanism, in which co-localization between substrate and invader is not mediated by base-pair complementarity, but by the presence of a target analyte (811). Both the invader and the substrate host a chemical modification or a DNA aptamer that can bind different epitopes on the analyte, whose presence thus causes the dimerization of the two DNA constructs. The latter then triggers the displacement of the incumbent. The released strand can finally be coupled to a downstream reaction cascade that signals the detection of the analyte, which has made TIDSD ideal for the development of DNA-based sensing platforms $(8-11)$.

The analyte-induced dimerization process exploited in TIDSD to transduce chemical signals mimics a widely conserved mechanism of cell biology (12). Indeed, a very diverse range of cellular processes are mediated by dimerization of soluble proteins induced by a specific analyte or ligand, including physiological cell death as mediated by the Bcl-2 family of proteins (13) and transcriptional control via ligand-activated transcription factors such as oestrogen and progesterone (14). Very often the sensing machinery exploiting ligand-induced dimerization 
is confined to biological membranes. Examples include Tyrosine Kinase receptors (15), the TGF- $\beta$ family of cytokines (16), and the T-cell receptor signalling pathways (17), all using a ligand to initiate transduction via the formation of homodimers or heterodimers of monomeric species (18). The variety of situations in which biological cells exploit this simple transduction mechanism follows from its applicability to a diverse range of targets, such as sterols, peptides, or even ions as in the case of cell adhesion mediation by E-cadherin induced by calcium (19). This remarkable versatility broadens the scope of biomimetic DNA-based sensing platforms relying on TIDSD, which have been shown to respond to targets such as streptavidin (8), various antibodies $(9,10)$, and the HIV viral capsid (11).

Motivated by the effectiveness and ubiquity of membrane-bound biological receptors activated by analyte-induced dimerization, here we explore the possibility of optimising the performance of DNA-based sensing devices relying on TIDSD by confining them to artificial lipid membranes. DNA constructs, here referred to as "receptors" to evoke the analogy with their biological counterparts, are tethered to the outer leaflet of liposomes (large unilamellar vesicles, LUVs) by means of cholesterol modifications (20-23). In the presence of a target analyte, the synthetic DNA-receptors dimerize and release an incumbent strand, triggering a downstream fluorescent response. For simplicity and generality, we consider target analytes consisting of ssDNA strands of various lengths, resulting in different degrees of co-localization in the dimerized receptors. We analyze the activation kinetics of the sensor through a combination of fluorimetry and coarse-grained Monte Carlo simulations, finding that confining the receptors to a membrane accelerates the process of strand release for all analytes. We show that the increased response rate follows from a greater rate of collision between the active sites where strand displacement is initiated.

The faster response of these membranescaffolded sensing platforms, as compared to solution-based circuits, is complemented by their comparatively leakage-free nature. Over a period of 70 hours, our membrane bound receptors demonstrated no discernible leakage, a feature the solution-based reaction networks do not share. These characteristics make the membrane-scaffolded networks excellent candidates for developing new diagnostic devices for generic biomarkers.

In addition to their biosensing potential, responsive liposomes are relevant in the context of bottom up synthetic biology as basic artificial cells (24, 25), along the lines of recent contributions by Rondelez and coworkers (26) and by De Greef and coworkers (27). Indeed, reaction networks on or within the these celllike units may be interfaced in a modular fashion with other DNA-nanotechnology elements to process information analogously to an in vivo signal cascade (28, 29), enabling complex life-like responses $(30,31)$.

\section{Results and Discussion}

\section{Implementation}

As illustrated in Fig. 1a (left), the TIDSD receptors are experimentally implemented as two DNA constructs. One receptor, labelled as $R_{1}$ (blue), is initially hybridized to an incumbent DNA molecule, $I$ (purple). The other DNA receptor, labelled as $R_{2}$ (red), features a singlestranded domain with the same sequence as $I$, which is thus thermodynamically capable of displacing the incumbent strand from receptor $R_{1}$, forming a receptor-receptor heterodimer.

The rate of incumbent displacement depends on the relative concentration of $R_{1} I$, and $R_{2}$, as strand-displacement reactions are known to be second order ( 7$)$. For freely diffusing receptors in solution at typical experimental concentrations (nanomolar range), the absence of any mechanisms co-localising the constructs makes incumbent displacement highly unlikely. The presence of the target analyte-strand $A$ (green in Fig. 1), which binds to two 15-base domains on $R_{1} I$ and $R_{2}$, induces their dimerization and substantially increases their local concentration. We term the resulting $A R_{1} I R_{2}$ structure 

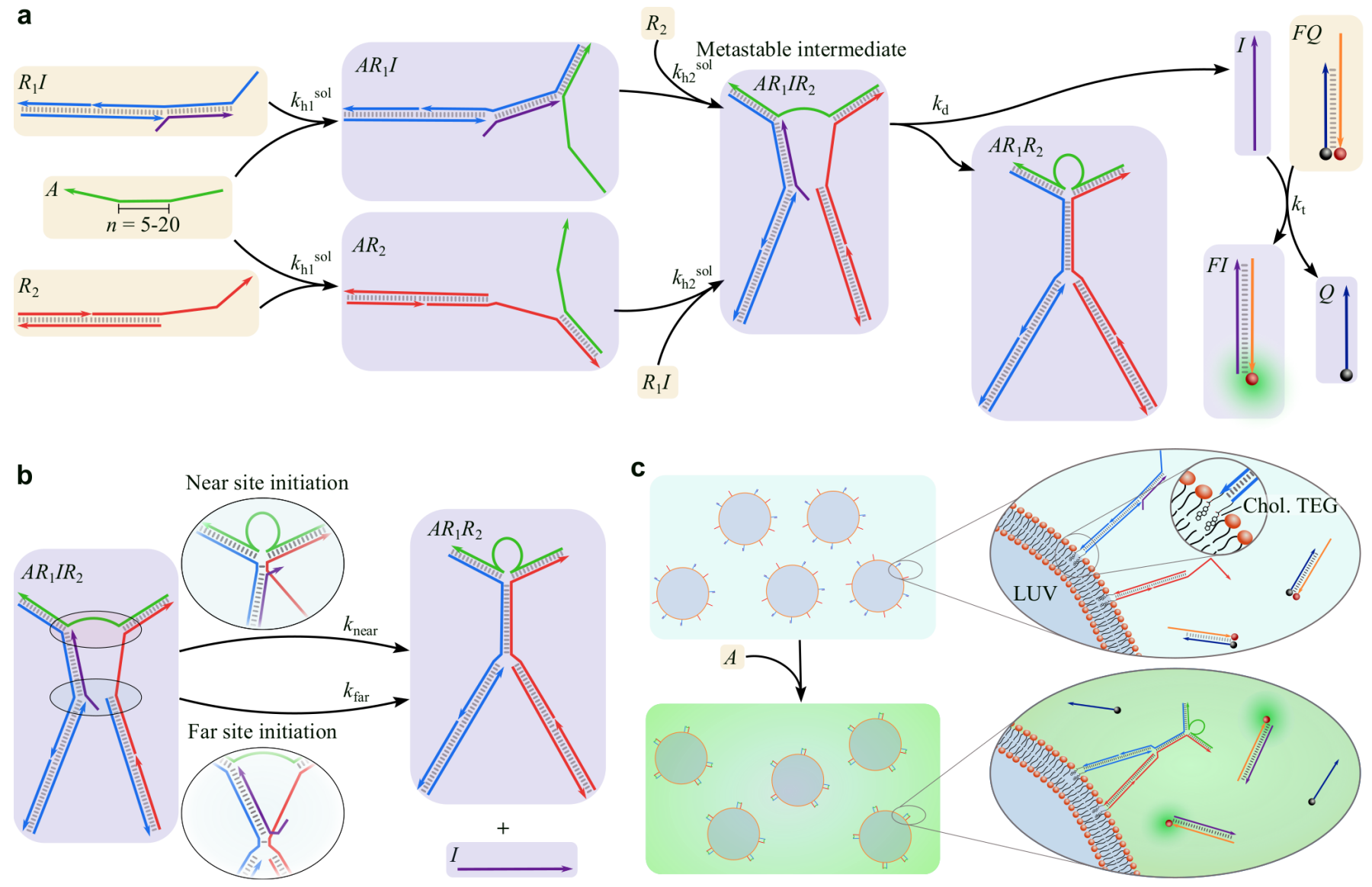

Figure 1: Implementation of DNA-based sensing platforms. a, Kinetic pathway leading to analyte detection for the solution-based system as described by Eqs. 1-6. From left to right, analyte strand $A$ leads to irreversible dimerization of receptor constructs $R_{1} I$ and $R_{2}$. Strand displacement results in the release of the incumbent strand $I$, which then displaces the quencher strand $Q$ from the reporter construct $F Q$, leading to a detectable fluorescent signal. b, Strand displacement can initiate at either end of the $R_{1} I$ duplex region, labelled as near and far active sites. c, Membranebased sensing platform. Receptors identical to those of the solution-based system (a) can be grafted to the outer surface of LUVs through a double-cholesterol-TEG modification.

the metastable intermediate (Fig. 1a, center), which is analagous to the three-strand intermediate in toehold-mediated strand displacement reactions (7), biotinylated DNA receptors dimerized by streptavidin (32), or DNA receptors dimerized by an antibody (9). The analyte-induced dimerization is effectively irreversible over experimental time scales.

The process of incumbent-strand displacement is expected to initiate with occasional fraying of the complementary bonds between $I$ and $R_{1}$, occuring most frequently at a termination of the double stranded region where base pairing is less stabilized by base stacking $(6,7,33)$. This transient opening creates an opportunity for the competing DNA strand $R_{2}$ to bind at that location instead, initiating a process known as branch migration $(34,35)$, where bonds are reversibly exchanged, here between $R_{1} I$ and $R_{1} R_{2}$. Branch migration is a stochastic process, often described as a $1 \mathrm{D}$ random walk in a rugged free-energy landscape $(6,7)$, that can either result in the system reverting to the metastable intermediate or progress until all possible $R_{1} R_{2}$ bonds are formed and the incumbent is released. As demonstrated by Fig. $1 \mathrm{~b}$ in our system, branch migration can initiate at either end of the double stranded region binding $R_{1}$ and $I$. We term the location of initiation nearest the analyte as the near active site, and 
the other as the far active site. Branch migration is fast compared to the timescales associated to its initiation, whose kinetics are the limiting factor in the overall TIDSD reaction (36).

The entropic gain associated to the release of the incumbent makes thermodynamically unfavourable the reverse reaction, which is also kinetically hindered by the absence of any mechanism co-localising $I$ with the $A R_{1} R_{2}$ construct. Furthermore, the freed incumbent $I$ is utilized to activate a fluorescent reporter circuit by displacing a quencher-bearing strand $(Q)$ from a fluorophore-bearing strand $(F)$ through toehold-mediated strand displacement (Fig. 1a, right). The fluorescence of duplex FI can thus be monitored over time to track the progression of the TIDSD reaction. Note that all duplexes in the system are at least 15 base-pair long, making them irreversible over experimental timescales unless the strands involved are displaced by analyte- or toehold-induced strand displacement reactions.

Two versions of the detection platform are experimentally implemented, one with the receptors $R_{1} I$ and $R_{2}$ freely dispersed in solution ("solution-based", Fig. 1a) and one in which the receptors are anchored to the outer surface of DOPC Large Unilamellar Vesicles (LUVs) ("membrane-based", Fig. 1c). Irreversible anchoring is achieved through a doublecholesterol modification applied to a duplex region on the opposite side of the receptors compared to the analyte anchoring point (Fig. 1c) (21, 22, 37, 38). Cholesterolized membranebound constructs are known to undergo free lateral diffusion across the fluid DOPC bilayer: even for large DNA origami tethered to a DOPC membrane by multiple cholesterol anchors, the diffusion coefficient of the con-

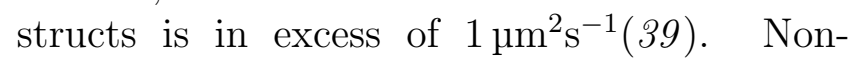
interacting (inert) DNA constructs are present on the LUVs along with the receptors, to produce sufficient steric repulsion and stabilize the liposomes against non-specific adhesion (21). Non-membrane-anchored receptors used for the solution-based platform are identical to the membrane-tethered ones, but lack the cholesterol moieties. All other components of the sensing circuit $(A, F Q)$ are dispersed in solution for both the solution-based and the membrane-based implementations.

Unless specified otherwise, for each experiment aimed at charaterizing the kinetics of the circuits' response samples initially contained $R_{1} I, R_{2}$ and $F Q$, each with bulk concentration of $20 \mathrm{nM}$, and $A$ at $10 \mathrm{nM}$. The same concentrations were used for both the solutionbased and membrane-based platforms. For the membrane-based system, the surface density of $R_{1} I$ and $R_{2}$ on the liposomes is kept constant at $50 \mathrm{\mu m}^{-2}$, and the concentration of LUVs tuned to achieve an initial bulk concentration of the receptors equal to $20 \mathrm{nM}$. After mixing all components at time $t=0$ we monitor the fluorescent emission of strand $F$ over time. Analytes of four different lengths are tested, with a number $n$ of free ssDNA thymine bases separating the two receptors equal to $5,10,15$ and 20 (Fig. 1a, left). All experiments are performed in near-physiological ionic strength (100 $\mathrm{mM} \mathrm{NaCl}+87 \mathrm{mM}$ glucose + TE buffer). See Methods section for details on sample preparation and measurement protocols and SI for full information on the structure of the DNA constructs (Fig. S1) and the sequences of their ssDNA components (Table S1).

\section{Kinetic Pathway}

The overall pathway is described by the following reactions, graphically summarized in Fig. 1a

$$
\begin{array}{cc}
R_{1} I+A \stackrel{k_{\mathrm{h} 1}^{\mathrm{mem} / \mathrm{sol}} \longrightarrow}{\longrightarrow} & A R_{1} I \\
R_{2}+A \stackrel{k_{\mathrm{h} 1}^{\mathrm{mem} / \mathrm{sol}}}{\longrightarrow} & A R_{2} \\
A R_{1} I+R_{2} \stackrel{k_{\mathrm{h} 2}^{\mathrm{mem} / \mathrm{sol}}}{\longrightarrow} & A R_{1} I R_{2} \\
R_{1} I+A R_{2} \stackrel{k_{\mathrm{h} 2}^{\mathrm{mem} / \mathrm{sol}}}{\longrightarrow} & A R_{1} I R_{2} \\
A R_{1} I R_{2} \stackrel{k_{\mathrm{d}}}{\longrightarrow} & A R_{1} R_{2}+I \\
I+F Q \stackrel{k_{\mathrm{t}}}{\longrightarrow} & F I+Q,
\end{array}
$$

where, Eqs. 1-4 describe the dimerization of the receptors induced by the analyte, Eq. 5 the strand-displacement process releasing $I$, and Eq. 6 the interaction of $I$ with the reporter 
a

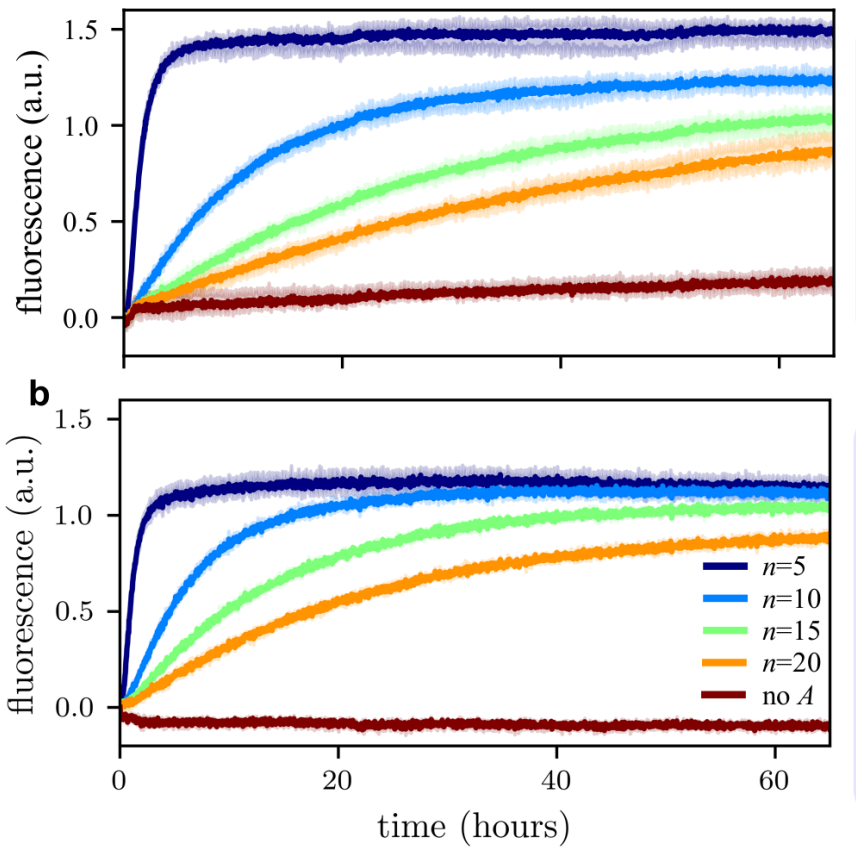

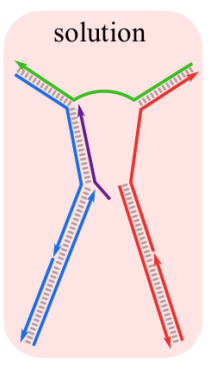

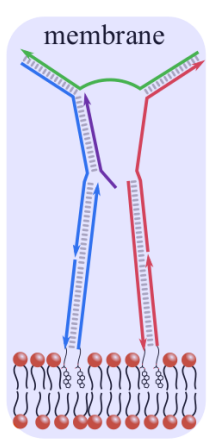

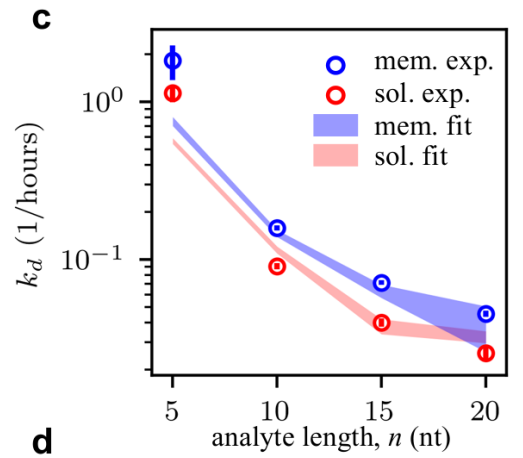

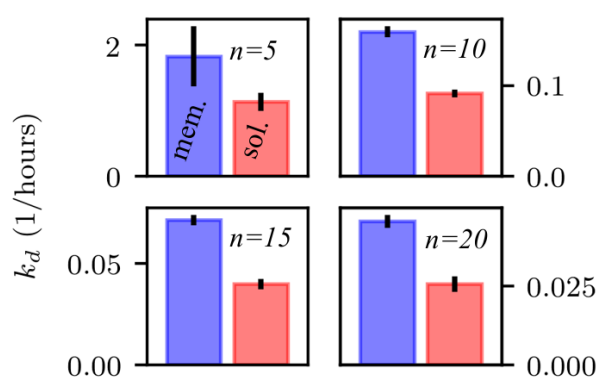

Figure 2: Membrane tethering speeds up analyte-sensing response. Fluorescent emission traces for analytes of different length $(n)$, and negative-control experiment in the absence of $A$ measured for the solution-based (a) and the membrane-based system (b). Errorbars are from the standard deviation over 8 repeats for the solution-based system, and 4 repeats for the membranebased system. c, Experimental incumbent-strand release rate $k_{\mathrm{d}}$ derived from the data in panels a and $\mathbf{b}$ (symbols) and best global fits based on Eqs. 7-9 and on the numerical (MC) estimates of the effective active-site concentrations $\rho_{\text {near }}$ and $\rho_{\text {far }}$ (shaded regions). Error bars on the experimental data correspond to one numerically-estimated standard deviation (see SI), those of the best fits are represented by the width of the curve and also correspond to one standard deviation. d, Direct comparison of $k_{\mathrm{d}}$ values between the membrane-tethered and solution based sensing platforms demonstrating a faster response rate in the former regardless on analyte length.

circuit.

The initial analyte-induced dimerization leading to the formation of the metastable intermediate is a two step process, in which the analyte hybridizes with the two receptors sequentially. Each of the two steps is a second-order reaction with rate $k_{\mathrm{h} 1}^{\mathrm{mem} / \mathrm{sol}}$ and $k_{\mathrm{h} 2}^{\mathrm{mem} / \mathrm{sol}}$, respectively, where the superscripts distinguish between he membrane-based and solution-based systems. Hybridization rates of free oligonucleotides are most often assumed to be in the region of $10^{6} \mathrm{M}^{-1} \mathrm{~s}^{-1}$ (7). However, a dependence on the viscosity and ionic strength of the solution has been reported in literature $(40,41)$. We measured the solution-phase hybridization rates experimen- tally (see Methods, SI, and Fig. S2a), obtaining $k_{\mathrm{h} 1}^{\mathrm{sol}}=k_{\mathrm{h} 2}^{\mathrm{sol}}=(1.00 \pm 0.05) \times 10^{5} \mathrm{M}^{-1} \mathrm{~s}^{-1}, \mathrm{com}^{-}$ patibly with values recorded at ionic strengths similar to those of our experiment (41).

To model analyte-induced dimerization events on the liposomes, we make a distinction between the hybridization of the freely-suspended analyte to the first membrane-anchored receptor, with rate $k_{\mathrm{h} 1}^{\mathrm{mem}}$, and the on-membrane hybridization of this to the second receptor, with rate $k_{\mathrm{h} 2}^{\mathrm{mem}}$. Previous reports found reduced hybridization rates for DNA oligonucleotides when their complementaries are tethered to solid substrates (41-43). In the absence of experimental estimates of the rates of hybridization of oligomers to complementary ones teth- 
ered to fluid membranes, we chose to use for $k_{\mathrm{h} 1}^{\mathrm{mem}}$ the same value measured for the bulk reactions $\left(k_{\mathrm{h} 1}^{\mathrm{mem}}=(1.00 \pm 0.05) \times 10^{5} \mathrm{M}^{-1} \mathrm{~s}^{-1}\right)$, being aware that this approximation provides an upper bound. Owing to the high effective concentration of tethered receptors on the membrane and their relatively high diffusivity (39) we assume the second step of the analyte-induced dimerization (Eqs. 3, 4) to be instantaneous as compared to all other processes, equivalent to $k_{\mathrm{h} 2}^{\mathrm{mem}} \rightarrow \infty$. As a result, and given the effective irreversibility of analytereceptor bonds, inter-LUV bridging events are kinetically inaccessible (21). Note that $k_{\mathrm{h} 1}^{\mathrm{mem} / \mathrm{sol}}$ and $k_{\mathrm{h} 2}^{\mathrm{mem} / \mathrm{sol}}$ are assumed to be independent on analyte length $n$.

The process of incumbent strand-displacement and release then follows first-order kinetics of rate constant $k_{\mathrm{d}}(8)$ (Eq. 5), while the toeholdmediated activation of the reporter circuit is best described as a second-order process with a rate constant $k_{\mathrm{t}}(6,7)$ (Eq. 6).

The rate $k_{\mathrm{t}}$ is conserved across implementations with different analyte length and free/membrane-bound receptors and could be experimentally estimated as $k_{\mathrm{t}}=(2.6 \pm 0.3) \times$ $10^{4} \mathrm{M}^{-1} \mathrm{~s}^{-1}$ (see Methods, SI, and Fig. S2b). Note that despite the long (7 nt) toehold between $I$ and $F$, the toeholding rate $k_{\mathrm{t}}$ in our system is still smaller than the free oligonucleotide hybridization rate $k_{\mathrm{h} 1 / 2}^{\mathrm{mem} / \mathrm{sol}}$, differently from previous reports where the two are found to converge for toeholds with length $\geq 6$ nt $(6,7)$. We argue that such a difference could be a consequence of the lower ionic strength of our experiments reducing the hybridization freeenergy of the invader-toehold interactions. Also strand $I$ can form a 3 base-pair stem-loop motif when isolated, which may reduce the rate of $Q$-strand displacement.

Having made estimates of $k_{\mathrm{h} 1}^{\mathrm{mem} / \mathrm{sol}}, k_{\mathrm{h} 2}^{\mathrm{mem} / \mathrm{sol}}$ and $k_{\mathrm{t}}$, we can then extract the incumbentdisplacement rate $k_{\mathrm{d}}$ by fitting the time dependent fluorescent signal, proportional to the concentration of un-quneched FI duplexes. Fitting is done using the numerical solutions of the coupled system of partial differential equations associated to the reaction pathway in Eqs. 1-6, as detailed in the SI.

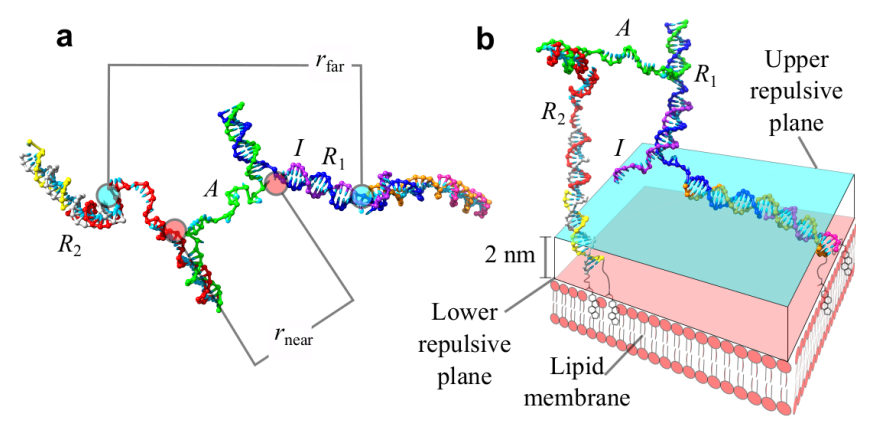

Figure 3: Coarse-grained modelling of the metastable intermediates enables estimation of the effective concentration of the active sites for initiation of strand displacement. Monte-Carlo simulation snapshots of the metastable intermediates for the solution-based (a) and the membrane-based (b) systems. Far and near active sites for initiation of strand displacement are highlighted in panel a along with their distance, sampled in the simulations. For the membrane-based system (b), the presence of the membrane is encoded by repulsive harmonic planes: all nucleotides are forced to be above the lower plane (pink) while the cholesterolized nucleotides are confined between the latter and the upper plane (light blue). Sketches of the lipid membrane and the cholesterol-TEG modifications are included for illustration purposes and are not part of the simulation. The cholesterolized strands have been given alternate colours from Fig. 1, to guide the eye in distinguishing them from the strands involved in the displacement process. Snapshots from simulation are visualized with UCSF Chimera (44).

\section{Effect of Membrane Scaffold on the Rate of Incumbent Strand Re- lease}

Fluorescent-emission traces in Fig. 2 demonstrate the effect of changing analyte length $n$ on the response rate of the system, for both the solution-based (Fig. 2a) and the membranebased implementations (Fig. 2b). In both cases, the response becomes monotonically slower with increasing $n$. Given that $k_{\mathrm{h} 1}^{\mathrm{mem} / \mathrm{sol}}, k_{\mathrm{h} 2}^{\mathrm{mem} / \mathrm{sol}}$ and $k_{\mathrm{t}}$ are independent on analyte length, the system's response is dictated by first-order rate 
of incumbent strand-displacement $k_{\mathrm{d}}$, which drops by almost two orders of magnitude between $n=5$ and $n=20$ (Fig. 2c).

The observed trends are readily explained as a consequence of the expected decrease in the effective concentration of active sites with increasing $n$, and are fully consistent with a qualitatively similar system studied in solution by Genot et al:: the remote-toehold (36). In this mechanism a toehold domain is separated from the location where branch migration initiates by a short single-stranded region; as this region increases in length the incumbent strand is released at a lower rate (36).

In Fig. 2c,d we compare the experimental incumbent-release rates of the solution-based and membrane-based implementations and, strikingly, we observe that the presence of the membrane results in larger values of $k_{\mathrm{d}}$ by a factor of between $1.3(n=5)$, and 1.8 $(n=20)$. Further, as the value of $k_{\mathrm{h} 1}^{\mathrm{mem}}$ utilized here represents an upper bound, the value of $k_{\mathrm{d}}$ determined for the membrane-based receptor can be seen as a lower bound, implying that membrane tethering may have an even larger accelerating effect on strand displacement than the one we estimate here.

Figure $2 \mathbf{a}$ and $\mathbf{b}$ also show fluorescence traces of negative-control experiments performed in the absence of the analyte $A$. A slight increase in fluorescence is observed for the solutionbased system, indicating a degree of leakage. Remarkably, no increase is observed for the membrane-based implementation. The comparatively leakage-free nature of the membranebased sensing platform is further discussed below.

\section{Coarse-Grained Numerical Simu- lations}

To rationalize the effect of membrane-induced confinement on the rate of incumbent strand displacement we perform Monte Carlo (MC) simulations on the metastable intermediates $A R_{1} I R_{2}$ for all the implementations of the system tested in experiments. We utilize the coarse-grained DNA representation oxDNA, which models each nucleotide as three beads: a backbone site, a $\pi$-stacking site, and a hydrogen bonding site, interacting through six potential energy terms $(45,46)$. The values for these potentials have been chosen through a top down parameterization approach to replicate thermodynamic and structural properties as determined by experiments $(45,46)$. Here we utilize the updated version of the model, oxDNA2 that explicitly accounts for screened Coulomb interactions and better approximates the geometry of the double-helix by introducing major and minor groves (47). For membranebound receptors, the presence of the membrane is modelled implicitly through harmonic constraints, as detailed in the Methods section and graphically summarized in Fig. 3a. Note that such a modelling choice results in fully flexible tethering points for the receptors that, in the absence of the mutual confinement induced by the analyte, would be able to pivot unimpeded around the (mobile) anchoring location. Although the assumption of free pivoting has been broadly employed when modelling DNA constructs anchored to synthetic membranes (21-23, 48), recent all-atom molecular dynamics simulations reported the possibility of preferential orientations, affected by multiple system-specific factors (49). Nonetheless, for the sake of generality and in the absence of data relative to our experimental implementation, we chose not to constrain the rotational motion of the receptors.

\section{Two Pathways for Displacement}

The first order rate constant $k_{\mathrm{d}}$ can be further described as a combination of rate constants representing displacement after initiation at the near and far active site

$$
k_{\mathrm{d}}=k_{\text {near }}+k_{\mathrm{far}},
$$

valid under the reasonable assumption that branch migration is highly unlikely to initiate simultaneously at both active sites.

In accordance with previous analysis, we conjecture that the rates of toeholdless displacement are proportional to the effective concen- 

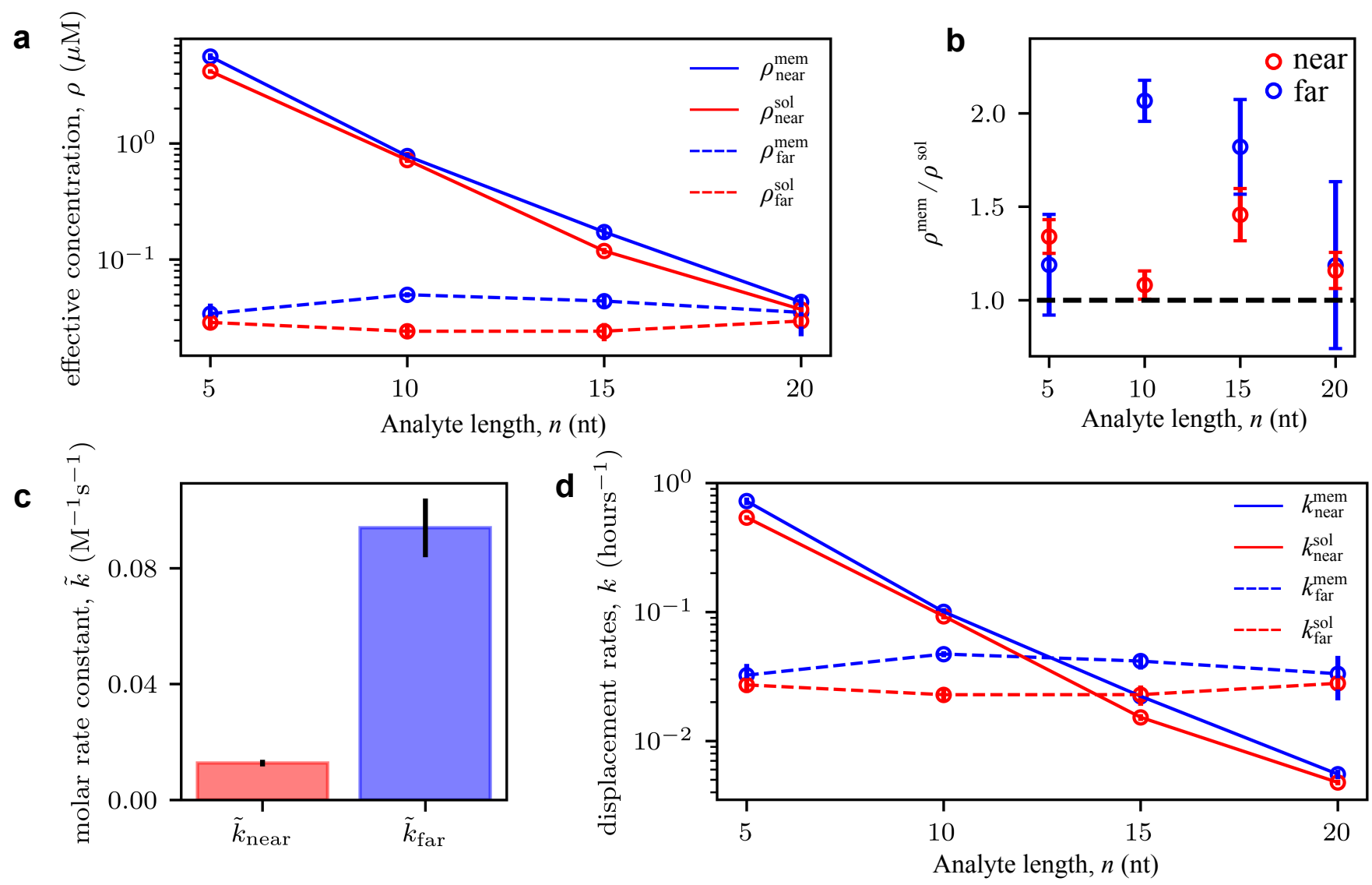

Figure 4: Effective concentration of active sites depends on membrane tethering and analyte length. a, Effective concentrations for the near active sites ( $\rho_{\text {near }}$, solid lines) and the far active sites ( $\rho_{\text {far }}$, dashed lines) as a function of analyte length $n$ as estimated from MC simulations. Data relative to the solution-based system are shown in red, those relative to the membrane-tethered system in blue. Errorbars are estimated as discussed in the Methods. b, Ratio between the nearand far-site effective concentrations shown in panel a. c, Second order rate constants $\tilde{k}_{\text {near }}$ and $\tilde{k}_{\text {far }}$ for initiation of strand displacement at the near and far active sites respectively, as determined from the fits in Fig. 2b (see Eqs. 7-9). d, Dependence of the corresponding first order rates $k_{\text {near }}$ and $k_{\text {far }}$ on $n$ (Eq. 7 ).

trations of active sites for branch-migration initiation (36) and define

$$
\begin{aligned}
k_{\text {near }} & =\tilde{k}_{\text {near }} \rho_{\text {near }} \\
k_{\text {far }} & =\tilde{k}_{\text {far }} \rho_{\text {far }},
\end{aligned}
$$

where $\rho_{\text {near/far }}$ are the effective concentrations of the near and far active sites and $\tilde{k}_{\text {near/far }}$ are the second-order rate coefficients.

MC simulations of the metastable intermediate are used to sample the distances $r_{\text {near }}$ and $r_{\text {far }}$ between the near and the far active sites (Fig. 3a), which we then use to estimate $\rho_{\text {near/far }}$ as detailed in the Methods.
Figure 4a summarizes the dependence of $\rho_{\text {near/far }}$ on analyte length $n$ for both membranetethered and free receptors. Interestingly, we observe that the effective concentration of the far active sites is nearly unaffected by $n$ while that of the near active sites drops exponentially with analyte length. Under the assumption that $\tilde{k}_{\text {near }}$ and $\tilde{k}_{\text {far }}$ are independent on analyte length, Eqs. 7, 8 and 9 suggest that a change in the likelihood of branch-migration initiation at the near active site is responsible for the observed $n$-dependence of $k_{\mathrm{d}}$ (Fig. 2c).

Figure $4 \mathbf{a}$, and more clearly Fig. 4b, demonstrate how membrane-tethered receptors ex- 
perience an increase in both $\rho_{\text {near }}$ and $\rho_{\text {far }}$ as compared to free constructs. This observation is fully consistent with the observed effect of membrane-tethering on $k_{\mathrm{d}}$, and thus offers a likely explanation for the accelerated response of the membrane-based sensing platform.

Rates of displacement are known to be contingent not only on the rate of initiation of branch migration, but heavily influenced by sequence identity, and ultimately the free-energy profile of branch migration (50). Since these free-energy profiles will certainly be different between the far and near displacement pathways, we expect $\tilde{k}_{\text {near }} \neq \tilde{k}_{\text {far }}$. In the assumption that $\tilde{k}_{\text {near }}$ and $\tilde{k}_{\text {far }}$ are independent on analyte length $n$ and unaffected by membrane tethering, we estimate the two coefficients by performing a global fitting of the experimentallydetermined values of $k_{\mathrm{d}}$ (Fig. 2b) and utilising the numerically-derived $\rho_{\text {near }}$ and $\rho_{\text {far }}$ (Fig. 4a). The fits reproduce reasonably well the experimental trends for $k_{\mathrm{d}}$ (Fig. 2b) and render estimates of the two rate coefficients that are markedly different in magnitude, with $\tilde{k}_{\text {far }} \sim 7 \tilde{k}_{\text {near }}$ (Fig. $4 \mathbf{c}$ ). The origin of the observed asymmetry may lay in the presence of a $5^{\prime}$ overhang in the incumbent strand $I$ at the location of the far active site. It has been previously observed that "inert tails" such as this overhang have the capacity of destabilising duplexes in moderate ionic strength conditions, such as those of the present experiment (51). We speculate that the overhang might aid the initiation of the branch migration process by lowering the free energy barrier for the formation of the first base-pairing bonds between the two receptors $R_{1}$ and $R_{2}$, for instance by enhancing fraying of the $R_{1} I$ duplex at the far active site.

Figure $4 \mathbf{d}$ shows the estimates of the first order rates of displacement for the near and far pathways, $k_{\text {near }}$ and $k_{\text {far }}$, as derived using Eqs. 8 and 9. As a result of the strong suppression of $\rho_{\text {near }}$ with increasing $n$ the two rates show a crossover point as a function of analyte length: if short analytes are used displacement is more likely to occur at the near site, while long analytes favour the far-site pathway.

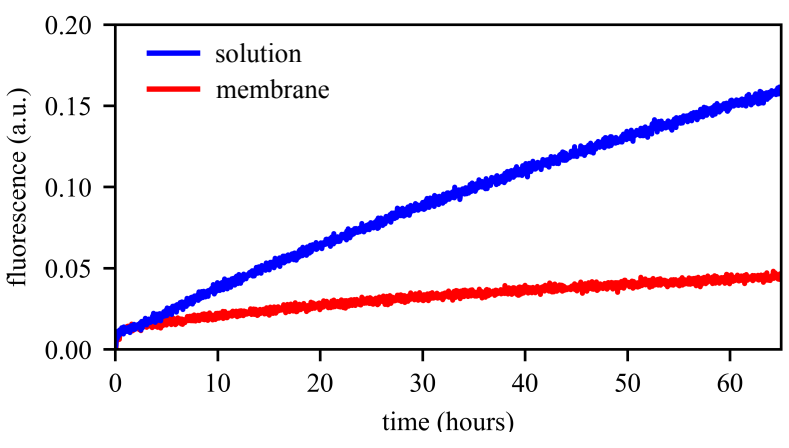

Figure 5: Membrane tethering suppresses leakage. Fluorescence-emission curves for negative-control leakage experiments performed in the absence of analyte strand $A$ at $5 \times$ concentration of all components for the solutionbased and membrane-based systems (100 nM). Note that for the membrane-based system a higher receptor concentration is achieved by increasing the concentration of liposomes while keeping the surface density of tethered receptors constant.

\section{Leakage reduction}

Toehold-less strand displacement reactions can over time result in the release of incumbent strands even in the absence of the analyte. This process, known as leakage, results in falsepositive fluorescent signals and may reduce the shelf-life of biosensing kits based on TIDSD.

As highlighted by the negative controls in Fig. 2a and b, in experimental conditions relevant to the results discussed this far leakage is limited over experimental time-scales (up to 70 hours), and even absent for the membranebased system.

To better assess the effect of membrane tethering on leakage, we thus perform negativecontrol experiments after increasing the concentration of $R_{1} I$ and $R_{2}$, along with that of the reporter element $F Q$, by a factor 5 resulting in an overall bulk concentration of $100 \mathrm{nM}$. Notably, for the membrane-based system, this is done by utilizing a $5 \times$ higher concentration of LUVs, while leaving unchanged the surface density of membrane-tethered receptors. As expected, the more concentrated systems display a non-negligible rate of leakage but still the membrane-based implementation appears to be 
significantly more resilient in comparison to the solution-based one (Fig. 5).

In our system, leakage can potentially occur via two independent pathways. One pathway initiates with a toehold-less strand displacement reaction between $R_{1} I$ and $R_{2}$ occurring without the formation of the metastable intermediate

$$
R_{1} I+R_{2} \leftrightarrow R_{1} R_{2}+I
$$

The released incumbent strand then activates the reporter circuit as described by Eq. 6. The second pathway is independent on the presence of $R_{2}$ and produces a fluorescent signal via a toehold-less strand displacement reaction between $R_{1} I$ and $F Q$

$$
R_{1} I+F Q \rightarrow R_{1} Q+F I .
$$

For the membrane-based system, the pathway described by Eq. 10 relies on interactions between tethered receptors, which may be influenced by factors such as membrane-induced orientational constraints (49), or steric hindrance produced by the presence of tethered inert DNA constructs. These effects might result in a slow down of the reaction in Eq. 10. Furthermore, we note that for the membrane-based platform analyte-less $R_{1} I-R_{2}$ reactions are most likely to occur between receptors tethered to the same liposome rather than across different LUVs. Thus we expect the reaction pathway in Eq. 10 to be weakly affected by increasing the overall bulk concentration of $R_{1} I$ and $R_{2}$, as long as their surface density on the liposomes remains constant.

Similarly, the leakage pathway described in Eq. 11 relies on interactions between a membrane-tethered $\left(R_{1} I\right)$ and a free construct $(F Q)$, which may be slower than the corresponding reactions occurring between two soluble elements (41-43), reducing leakage rate.

\section{Conclusion}

Through a series of proof-of-concept experiments and computer simulations, we have demonstrated that membrane scaffolding provides a mechanism to enhance TIDSD, both ac- celerating responsiveness and suppressing leak reactions.

Membrane tethering of the sensing DNA constructs increases the effective concentration of the active sites for initiation of branch migration, which leads to a higher rate of incumbentstrand displacement and ultimately to an overall faster response of the system to the presence of the target analyte. The increase in response rate occurs regardless on analyte size, and thus on the degree of confinement it imposes between the two receptors.

Receptor-functionalized liposomes demonstrate substantially more stability than their free in solution analogues, being leak free on time periods of at least 70 hours.

These factors make liposome scaffolded TIDSD optimal for the development of new diagnostic tools in a clinical setting $(8-11)$. The receptor functionalized liposomes can also be regarded as simple cell-like agents able to exchange information with the local chemical environment, a starting point for the development of synthetic cell consortia in the context of bottom-up synthetic biology $(26,27)$.

\section{Methods}

\section{Materials}

\section{DNA}

Sequences of the reporter circuit were taken from Ref. (8). Remaining sequences were reverse-engineered to interact with that reporter circuit using the NUPACK design tool (52). The sequences of all the strands used are shown in SI, Table S1, while the detailed structure is shown in Fig. S1. 5'-cholesterylmodified DNA strands were purchased from Eurogentec (Liege, Belgium) with High Performance Liquid Chromatography (HPLC) purification. All other DNA strands were purchased from Integrated DNA Technologies (Coralville, Iowa, United States) also purified by HPLC. Strand $F$ was modified with 6-FAM (Fluoroscein). $Q$, was modified with Iowa Black. 


\section{Lipids and Buffers}

Sodium chloride (BioUltra, >99.5\%), D$(+)$-Glucose $\quad(>99.5 \%), \quad 100 \times \quad$ TrisEDTA buffer and Sucrose were purchased from Sigma-Aldrich. 1,2-dioleoyl-sn-glycero-3phosphocholine (DOPC, >99\%) was purchased from Avanti Polar Lipids Inc (Alabaster, Alabama, USA). Texas-Red tagged 1,2-dihexadecanoyl-sn-glycero-3-p hosphoethanolamine, triethylammonium salt (TXDHPE) was purchased from ThermoFisher Scientific (Waltham, Massachusetts, USA).

\section{Experimental Methods}

\section{DNA Handling}

DNA was shipped lyophilized, and was reconstituted to a concentration of approximately $100 \mu \mathrm{M}$ in TE buffer $(1 \mathrm{mM}$ EDTA, $10 \mathrm{mM}$ Tris, $\mathrm{pH}$ 8.0). Buffers were filtered through $0.22 \mu \mathrm{m}$ pore polyethersulfone filters (Millex) prior to use. Absorbance of the stock solutions at $260 \mathrm{~nm}$ was evaluated by UV-vis spectrophotometry on a Thermo Scientific NanoDrop 2000. The concentrations of the stock solutions were evaluated by the ratio of the absorbance at $260 \mathrm{~nm}$ to the extinction coefficient of the DNA. Stock solutions were subsequently stored frozen at $-20^{\circ} \mathrm{C}$.

\section{DNA Hybridization}

Assembly of multistranded DNA constructs, namely $R_{1} I, R_{2}, F Q$ and inert constructs (see SI, Fig. S1 and Table S1), was facilitated by thermal annealing. For each construct all single-stranded components were combined at equal concentrations of $2 \mu \mathrm{M}$ in $100 \mathrm{mM} \mathrm{NaCl}$ TE buffer, with the only exception of $F Q$, where a $50 \%$ excess of $Q$ was used ( $F-Q$ molar ratio of 2:3). The annealing process was carried out on a Techne TC-512 thermocycler. The strands were heated to $90^{\circ} \mathrm{C}$ for 5 minutes to ensure all DNA was melted, and cooled steadily to $4^{\circ} \mathrm{C}$ over three hours.

\section{LUV Synthesis}

DOPC Large Unilamellar Vesicles (LUVs) were formed via extrusion, as detailed in previous reports (53). Briefly, $220 \mathrm{\mu l}$ of DOPC solution in chloroform $\left(25 \mathrm{mg} \mathrm{ml}^{-1}\right.$, Avanti Polar Liquids) were added to a glass vial and desiccated under vacuum. The DOPC film then was rehydrated in $500 \mathrm{\mu l}$ of $300 \mathrm{mM}$ sucrose in MilliQ water by vortexing, and freeze thawed under liquid nitrogen 4 times. The resultant suspension was extruded using a mini extruder kit (Avanti Polar Liquids) through a polycarbonate membrane pore (Whatman) of pore size $1.0 \mathrm{\mu m}$. LUVs were stored at room temperature and used within 3 days.

\section{Functionalising LUVs with DNA}

LUVs were functionalized by incubation with cholesterolized DNA constructs in a solution of $100 \mathrm{mM} \mathrm{NaCl}+87 \mathrm{mM}$ glucose TE buffer for 24 hours at room temperature. The osmolarity of this solution was chosen to match the osmolarity of the liposomes to prevent osmoticshock-induced rupture. During incubation, the concentration of lipids and DNA was kept to $5 \times$ the eventual concentration in the final samples. The ratio of LUVs to cholesterolized receptors was chosen for the eventual surface concentration of each receptor type, $R_{1}$ and $R_{2}$, to be one per $2000 \mathrm{~nm}^{2}$ (surface density $50 \mathrm{\mu m}^{-2}$ ). Calculation of the surface density of DNA was performed by assuming $100 \%$ efficiency of LUV synthesis, and an average area per DOPC headgroup of $72.5 \AA$ (54). Additional "inert" cholesterolized double stranded DNA (dsDNA) was added to increase the total surface concentration of DNA to one strand every $500 \mathrm{~nm}^{2}$ (including $R_{1}, R_{2}$ and inert strands). A higher surface coverage was required to provide sufficient steric repulsion between the liposomes and prevent their aggregation as confirmed by confocal microscopy (21). The design of the inert strands is shown in the SI, Fig. S1.

To verify that incubation leads to adherence of the cholesterolized nanostructures to the membrane, the protocol was repeated using LUVs made fluorescent through a $10 \%$ molar fraction of TX-DHPE. After the 24-hour inclubation, 
the DNA intercalating dye Sybr Safe (SigmaAldrich) was added to the sample. Fluorescencent signal was measured at $596 \mathrm{~nm} / 616 \mathrm{~nm}$ (excitation / emission, Texas Red) and $485 \mathrm{~nm}$ / $515 \mathrm{~nm}$ (Sybr Safe) using a Cary Eclipse fluorimeter. The solution was then centrifuged at 8000 rcf for 30 minutes to sediment the vesicles before measuring the fluorescence of the supernatant. We observed 90\% cosegreation of the Sybr Safe signal (corresponding to DNA) with the Texas Red signal (corresponding to the LUVs), indicating that that vast majority of the DNA was indeed grafted to the liposomes. To remove any DNA not adhering to the vesicles, after the incubation process functionalized vesicles are centrifuged for 30 minutes at $1000 \mathrm{rcf}$, the eluent discarded and replaced with fresh buffer. Vesicles were then resuspended by gently pipetting up and down until the pellet had vanished.

\section{Fluorescence Kinetic Assays}

For both the solution-based and the membranebased sensors, reactions aimed at characterizing the kinetics of their response were performed at $100 \mathrm{\mu l}$ volume in a 96 well plate (Grenier 96 well F-bottom) in a solution of $100 \mathrm{mM} \mathrm{NaCl}, 87 \mathrm{mM}$ glucose TE buffer. For the solution-based system, samples initially contained $20 \mathrm{nM} F Q$, $20 \mathrm{nM} R_{1} I, 20 \mathrm{nM} R_{2}, 10 \mathrm{nM} A, 10 \mathrm{nM} Q, 1 \mu \mathrm{M}$ 20 nt-long polyT. The excess $Q$ results from the concentration mismatch of the two components in the preparation of $F Q$ and does not affect the kinetic response of the system. Samples for testing the membrane-based platform contained the same components, except that $R_{1} I$ and $R_{2}$ were replaced by a suitable concentration of receptor-functionalized vesicles, calculated to achieve the same overall concentration of receptors of the free-receptor case. The purpose of the polyT was to reduce non-specific adsorption of DNA constructs to the walls of the well plate.

Fluorescence was monitored at $485 \mathrm{~nm} / 520 \mathrm{~nm}$ for 70 hours with a Fluostar Omega (BMG Labtech), using a cycle time of $120 \mathrm{~s}$, with 100 flashes per well (bottom optic), and 1 second of $100 \mathrm{~Hz}$ vibration between cycles to ensure a well mixed system. The measurements were started within two minutes of mixing all the components. Within the first few minutes of control experiments with only $20 \mathrm{nM} F Q$, there is a small $(\sim 0.1)$ decline in observed fluorescence, followed by no change over the subsequent 70 hours. To control for this effect, we reduce each of the time varying fluorescence traces by a control experiment containing only $20 \mathrm{nM} F Q$, and $1 \mu \mathrm{M}$ polyT. Initially there is substantial nonuniform fluorescence across different wells. To account for this, we reduce the fluorescence traces by the first measured value. Measurements were repeated 8 times for each analyte length for the solution-based system and 4 times for the membrane-based system.

\section{Determining $k_{\mathrm{h} 1 / 2}^{\mathrm{mem} / \mathrm{sol}}$ and $k_{\mathrm{t}}$}

The bulk oligonucleotide-hybridization rate used to approximate $k_{\mathrm{h} 1 / 2}^{\mathrm{mem} / \mathrm{sol}}$ was determined through a dedicated absorbance experiment. The second-order rate constant $k_{\mathrm{t}}$ describing the activation of the reporter circuit was determined though positive-control samples with $I$ and $F Q$. Full details are reported in the SI text and Fig. S2.

\section{Extracting $k_{\mathrm{d}}$}

Using the estimates of $k_{\mathrm{h} 1 / 2}^{\mathrm{mem} / \mathrm{sol}}$ and $k_{\mathrm{t}}$, we determine $k_{\mathrm{d}}$ by fitting experimental fluorescence curves for each of the tested conditions with the numerical outcomes of Eqs. 1-6, under the assumption that the fluorescent signal is proportional to the concentration of the FI (Eq. 6) Uncertainty on the $k_{\mathrm{d}}$ parameter was extracted via a bootstrap resampling mechanism. Full details are provided in the SI.

\section{Monte Carlo Simulations}

\section{Encoding the Effect of the Membrane}

oxDNA has been parameterized only for DNA. To examine the effect of confinement on the distribution of conformations of the metastable intermediate for the membrane-bound case, the membrane was modelled implicitly through 
harmonic constraints. A lower repulsive plane (pink in Fig. 3b) confines all particles to be above it, encoding the steric effect of the membrane. Two nanometers above this, an upper repulsive plane (light blue in Fig. 3b) is applied to the four cholesterolized nucleotides, encoding for the presence of the TEG-cholesterol linker and limiting diffusion of the cholesterolized strands away from the membrane. To identify if the simulation results were contingent on the distance between the planes, simulations were performed also using a constraint of $1 \mathrm{~nm}$, which resulted in negligable differences.

\section{Initialization}

The following mechanism was used to generate structures corresponding to the metastable intermediates for the cases of membrane-bound and free receptors. DNA strands were first initialized at random locations then, via a custommade Python script, harmonic traps were introduced between nucleotides which would be paired in the the final structure, forcing the system to relax into the sought configuration. Without these traps, which were removed after initialization, the computation time taken for the strands to form the expected structure is prohibitively long. This initial assembly was performed using Virtual Move Monte Carlo (VMMC) (55), with maximum cluster size equal to the entire system. During initialization, the monovalent salt concentration was set artificially high to minimize repulsion and accelerate Watson-Crick bond formation. Typical configurations of the metastable intermediates are illustrated in Fig. 2.

\section{Running Simulations}

Once formed, the metastable intermediate was sampled using VMMC (55). The temperature of the system was set to $27^{\circ} \mathrm{C}$, and a monovalent salt concentration set to $0.1 \mathrm{M}$, in line with experiments. The salt concentration affects only screening of the repulsion between the phosphate backbones, under a mean field DebyeHuckel treatment. To enhance sampling of the regions of the configuration space where the ac- tive sites are close, simulations were biased. For each metastable intermediate, separate simulations were run with a biasing potential between the near active sites and between the far active sites, with form $U_{\text {bias }}=r^{2} \cdot 0.0692 k_{\mathrm{B}} T$, where $r=r_{\text {far }}$ or $r_{\text {near }}$. An ensemble of 64 metastable intermediates for each system were simulated for 10 hours from the same initial configuration. Snapshots of the system were stored every 100 cluster moves. The time taken for equilibration and the statistical inefficiency of sampling the observable, $r_{\text {near/far }}$ were estimated through a recently developed method implemented in the Python package pymbar (56). The number of independent samples acquired in the simulation varied between 1,000 and 16,000 depending on the system in question.

\section{Postprocessing}

Trajectories were postprocessed using custom Python scripts to extract the distances between the actives sites, $r_{\text {near/far }}$ (Fig. 3a). The probability for the distances between the active sites to be within a given limit $r_{\max }$ was then estimated as

$$
p\left(r_{\text {near } / \text { far }}<r_{\max }\right)=\frac{\int_{0}^{r_{\text {max }}} p(r)_{\pi} e^{U_{\text {bias }}(r) \beta} \mathrm{d} r}{\int_{0}^{\infty} p(r)_{\pi} e^{U_{\text {bias }}(r) \beta} \mathrm{d} r},
$$

where $p\left(r_{\text {near/far }}\right)_{\pi}$ is the biased probability, extracted directly for the distribution of active site distance (for brevity we omit the subscript near/far in the right hand side of Eq. 12). To estimate the bootstrapped uncertainties of $p\left(r_{\text {near/far }}<r_{\max }\right)$, the subsampled probability distribution was resampled (50 replacements), and for each resampling, $p\left(r_{\text {near/far }}<r_{\max }\right)$ acquired through the bias correction procedure. The uncertainty as used here is from the standard deviation of the resampled values. The probability $p\left(r_{\text {near/far }}<r_{\max }\right)$ was then converted to an effective concentration of the active sites as

$$
\begin{aligned}
\rho_{\text {near }} & =\frac{p\left(r_{\text {near }}<r_{\max }\right)}{V_{\max }} \\
\rho_{\text {far }} & =\frac{p\left(r_{\text {far }}<r_{\max }\right)}{V_{\max }},
\end{aligned}
$$


where $V_{\max }=4 / 3 \pi r_{\max }^{3}$ and $r_{\max }$ is the minimum distance where our model is accurate, set to $3.5 \mathrm{~nm}$. Using the numerically-generated values of $\rho_{\text {near }}$ and $\rho_{\text {far }}$ for all receptor types and analyte lengths, in combination with Eqs. 7, 8 and 9 we obtain a two-parameter family of curves in which $\tilde{k}_{\text {far }}$ and $\tilde{k}_{\text {near }}$ are unknown, and can be determined by fitting this family of curves to the 12 datasets of experimental rates $k_{\mathrm{d}}, 4$ for membrane bound systems, 8 for solution based systems (corresponding to independent repeats). Fitting is performed using minimization of $\chi^{2}$, where the uncertainties for each datapoint are given by combined experimental uncertainties in $k_{\mathrm{d}}$ and simulation uncertainties in $\rho_{\text {near }}$ and $\rho_{\text {far }}$.

Acknowledgement LDM and PC, acknowledge support from the EPSRC Programme Grant CAPITALS number EP/J017566/1. LDM acknowledges support from the Leverhulme Trust and the Isaac Newton Trust through an Early Career Fellowship (ECF2015-494) and from the Royal Society through a University Research Fellowship (UF160152). JMT and RAB acknowledge support from the EPSRC CDT in Nanoscience and Nanotechnology (NanoDTC), grant number EP/L015978/1. WTK acknowledges support from an EPSRC DTP studentship. This work was performed using the Darwin Supercomputer of the University of Cambridge High Performance Computing Service (http://www.hpc.cam.ac.uk/), provided by Dell Inc. using Strategic Research Infrastructure Funding from the Higher Education Funding Council for England and funding from the Science and Technology Facilities Council.

\section{Supporting Information Available:} Structure of the DNA constructs, DNA sequences, estimation of kinetic rates and numerical methods. This material is available free of charge via the Internet at http: //pubs.acs.org/.

\section{References}

(1) Rothemund, P. W. K. (2006) Folding DNA to create nanoscale shapes and patterns. Nature 440, 297-302.

(2) Seeman, N. C. (2007) An overview of structural DNA nanotechnology. Mol. Biotech. 37, 246-57.

(3) Seeman, N. C., and Sleiman, H. F. (2017) DNA nanotechnology. Nat. Rev. Mater. 3, 17068 .

(4) Brady, R. A., Brooks, N. J., Cicuta, P., and Di Michele, L. (2017) Crystallization of Amphiphilic DNA C-Stars. Nano Lett. 17, 3276-3281.

(5) Brady, R. A., Brooks, N. J., Foderà, V., Cicuta, P., and Di Michele, L. (2018) Amphiphilic-DNA Platform for the Design of Crystalline Frameworks with Programmable Structure and Functionality. J. Am. Chem. Soc. 140, 15384-15392.

(6) Srinivas, N., Ouldridge, T. E., Šulc, P., Schaeffer, J. M., Yurke, B., Louis, A. A., Doye, J. P. K., and Winfree, E. (2013) On the biophysics and kinetics of toeholdmediated DNA strand displacement. $\mathrm{Nu}$ cleic Acid Res. 41, 10641-10658.

(7) Zhang, D. Y., and Winfree, E. (2009) Control of DNA Strand Displacement Kinetics Using Toehold Exchange. J. Am. Chem. Soc. 131, 17303-17314.

(8) Li, F., Tang, Y., Traynor, S. M., Li, X.F., and Le, X. C. (2016) Kinetics of Proximity-Induced Intramolecular DNA Strand Displacement. Anal. Chem. 88, 8152-8157.

(9) Engelen, W., Meijer, L. H. H., Somers, B., de Greef, T. F. A., and Merkx, M. (2017) Antibody-controlled actuation of DNAbased molecular circuits. Nat. Commun. 8,14473 .

(10) Ranallo, S., Prévost-Tremblay, C., Idili, A., Vallée-Bélisle, A., and Ricci, F. 
(2017) Antibody-powered nucleic acid release using a DNA-based nanomachine. Nat. Commun. 8, 15150.

(11) Porchetta, A., Ippodrino, R., Marini, B., Caruso, A., Caccuri, F., and Ricci, F. (2018) Programmable Nucleic Acid Nanoswitches for the Rapid, Single-Step Detection of Antibodies in Bodily Fluids. J. Am. Chem. Soc. 140, 947-953.

(12) Klemm, J. D., Schreiber, S. L., and Crabtree, G. R. (1998) Dimerization as a regulatory mechanism in signal transduction. Annu. Rev. Immunol. 16, 569-592.

(13) Reed, J. C., Miyashita, T., Krajewski, S., Takayama, S., Aime-Sempe, C., Kitada, S., Sato, T., Wang, H. G., Harigai, M., Hanada, M., Krajewska, M., Kochel, K., Millan, J., and Kobayashi, H. (1996) Bcl-2 family proteins and the regulation of programmed cell death in leukemia and lymphoma. Cancer. Treat. Res. 84, 31-72.

(14) Tsai, M.-j., and O'Malley, B. W. (1994) Molecular mechanisms of action of steroid / thyroid receptor superfamily members. Annu. Rev. Biochem. 63, 451-486.

(15) Heldin, C.-H. (1995) Dimerization of cell surface receptors in signal transduction. Cell 80, 213-223.

(16) Massagué, J. (1996) TGF $\beta$ signaling: Receptors, transducers, and mad proteins. Cell 85, 947-950.

(17) Weiss, A., and Littman, D. R. (1994) Signal transduction by lymphocyte antigen receptors. Cell 76, 263-274.

(18) Sako, Y., Minoghchi, S., and Yanagida, T. (2000) Single-molecule imaging of EGFR signalling on the surface of living cells. Nat. Cell Biol. 2, 168-172.

(19) Nagar, B., Overduin, M., Ikura, M., and Rini, J. M. (1996) Structural basis of calcium-induced E-cadherin rigidification and dimerization. Nature 380, 360-364.
(20) Beales, P. A., and Kyle Vanderlick, T. (2007) Specific binding of different vesicle populations by the hybridization of membrane-anchored DNA. J. Phys. Chem. A 111, 12372-12380.

(21) Parolini, L., Kotar, J., Di Michele, L., and Mognetti, B. M. (2016) Controlling Self-Assembly Kinetics of DNAFunctionalized Liposomes Using Toehold Exchange Mechanism. ACS Nano 10, 2392-2398.

(22) Bachmann, S. J., Kotar, J., Parolini, L., Saric, A., Cicuta, P., Di Michele, L., and Mognetti, B. M. (2016) Melting transition in lipid vesicles functionalised by mobile DNA linkers. Soft Matter 12, 7804-7817.

(23) Parolini, L., Mognetti, B. M., Kotar, J., Eiser, E., Cicuta, P., and Di Michele, L. (2015) Volume and porosity thermal regulation in lipid mesophases by coupling mobile ligands to soft membranes. Nat. Commun. 6, 5948.

(24) Trantidou, T., Friddin, M., Elani, Y., Brooks, N. J., Law, R. V., Seddon, J. M., and Ces, O. (2017) Engineering Compartmentalized Biomimetic Micro- and Nanocontainers. ACS Nano 11, 65496565 .

(25) Buddingh', B. C., and van Hest, J. C. M. (2017) Artificial Cells: Synthetic Compartments with Life-like Functionality and Adaptivity. Acc. Chem. Res. 50, 769-777.

(26) Gines, G., Zadorin, A. S., Galas, J. C., Fujii, T., Estevez-Torres, A., and Rondelez, Y. (2017) Microscopic agents programmed by DNA circuits. Nat. Nano. 12, 351-359.

(27) Joesaar, A., Yang, S., Bogels, B., van der Linden, A., Kumar, P., Dalchau, N., Phillips, A., Mann, S., and de Greef, T. (2019) Distributed DNA-based Communication in Populations of Synthetic Protocells. Nat. Nano. doi.org/10.1038/s41565019-0399-9. 
(28) Montagne, K., Plasson, R., Sakai, Y., Fujii, T., and Rondelez, Y. (2011) Programming an in vitro DNA oscillator using a molecular networking strategy. Mol. Sys. Biol. 7, 466.

(29) Padirac, A., Fujii, T., and Rondelez, Y. (2012) Bottom-up construction of in vitro switchable memories. Proc. Natl. Acad. Sci. USA 109, E3212-20.

(30) Amir, Y., Ben-Ishay, E., Levner, D., Ittah, S., Abu-Horowitz, A., and Bachelet, I. (2014) Universal computing by DNA origami robots in a living animal. Nat. Nano. 9, 353-357.

(31) Groves, B., Chen, Y.-J., Zurla, C., Pochekailov, S., Kirschman, J. L., Santangelo, P. J., and Seelig, G. (2016) Computing in mammalian cells with nucleic acid strand exchange. Nat. Nano. 11, 287-294.

(32) Li, F., Zhang, H., Wang, Z., Li, X., Li, X.F., and Le, X. C. (2013) Dynamic DNA Assemblies Mediated by Binding-Induced DNA Strand Displacement. J. Am. Chem. Soc. 135, 2443-2446.

(33) Zgarbová, M., Otyepka, M., Šponer, J., Lankaš, F., and Jurečka, P. (2014) Base Pair Fraying in Molecular Dynamics Simulations of DNA and RNA. J. Chem. Theory Comput. 10, 3177-3189.

(34) Radding, C. M., Beattie, K. L., Holloman, W. K., and Wiegand, R. C. (1977) Uptake of homologous singlestranded fragments by superhelical DNA: IV. Branch migration. J. Mol. Biol. 116, 825-839.

(35) Green, C., and Tibbetts, C. (1981) Reassociation rate limited displacement of DNA strands by branch migration. $\mathrm{Nu}$ cleic Acid Res. 9, 1905-18.

(36) Genot, A. J., Zhang, D. Y., Bath, J., and Turberfield, A. J. (2011) Remote Toehold: A Mechanism for Flexible Control of DNA Hybridization Kinetics. J. Am. Chem. Soc. 133, 2177-2182.
(37) Pfeiffer, I., and Höök, F. (2004) Bivalent Cholesterol-Based Coupling of Oligonucletides to Lipid Membrane Assemblies. J. Am. Chem. Soc. 126, 10224-10225.

(38) Beales, P. A., and Vanderlick, T. K. (2009) Partitioning of Membrane-Anchored DNA between Coexisting Lipid Phases. J. Phys. Chem. B 113, 13678-13686.

(39) Czogalla, A., Franquelim, H. G., and Schwille, P. (2016) DNA Nanostructures on Membranes as Tools for Synthetic Biology. Biophys. J. 110, 1698-1707.

(40) Wetmur, J. G., and Davidson, N. (1968) Kinetics of renaturation of DNA. J. Mol. Biol. 31, 349-370.

(41) Gao, Y., Wolf, L. K., and Georgiadis, R. M. (2006) Secondary structure effects on DNA hybridization kinetics: a solution versus surface comparison. $\mathrm{Nu}$ cleic Acid Res. 34, 3370-3377.

(42) Henry, M. R., Wilkins Stevens, P., Sun, J., and Kelso, D. M. (1999) Real-Time Measurements of DNA Hybridization on Microparticles with Fluorescence Resonance Energy Transfer. Anal. Biochem. 276, 204-214.

(43) Sekar, M. M. A., Bloch, W., and St John, P. M. (2005) Comparative study of sequence-dependent hybridization kinetics in solution and on microspheres. Nucleic Acid Res. 33, 366-375.

(44) Pettersen, E., Goddard, T., Huang, C. C., Couch, G., Greenblatt, D., E.C., M., and T.E., F. (2004) UCSF Chimera-a visualization system for exploratory research and analysis. J. Comput. Chem. 25, 160512 .

(45) Ouldridge, T. E., Louis, A. A., and Doye, J. P. K. (2010) DNA Nanotweezers Studied with a Coarse-Grained Model of DNA. Phys. Rev. Lett. 104, 178101. 
(46) Ouldridge, T. E., Louis, A. A., and Doye, J. P. K. (2011) Structural, mechanical, and thermodynamic properties of a coarse-grained DNA model. J. Chem. Phys. 134, 085101.

(47) Snodin, B. E. K., Randisi, F., Mosayebi, M., Šulc, P., Schreck, J. S., Romano, F., Ouldridge, T. E., Tsukanov, R., Nir, E., Louis, A. A., and Doye, J. P. K. (2015) Introducing improved structural properties and salt dependence into a coarse-grained model of DNA. J. Chem. Phys. 142, 234901.

(48) Shimobayashi, S. F., Mognetti, B. M., Parolini, L., Orsi, D., Cicuta, P., and Di Michele, L. (2015) Direct measurement of DNA-mediated adhesion between lipid bilayers. Phys. Chem. Chem. Phys. 17, 15615-15628.

(49) Arnott, P. M., Joshi, H., Aksimentiev, A., and Howorka, S. (2018) Dynamic Interactions between Lipid-Tethered DNA and Phospholipid Membranes. Langmuir 34, 15084-15092.

(50) Machinek, R. R. F., Ouldridge, T. E., Haley, N. E. C., Bath, J., and Turberfield, A. J. (2014) Programmable energy landscapes for kinetic control of DNA strand displacement. Nat. Commun. 5, 5324

(51) Di Michele, L., Mognetti, B. M., Yanagishima, T., Varilly, P., Ruff, Z., Frenkel, D., and Eiser, E. (2014) Effect of Inert Tails on the Thermodynamics of DNA Hybridization. J. Am. Chem. Soc. 136, 6538-6541.

(52) Zadeh, J. N., Steenberg, C. D., Bois, J. S., Wolfe, B. R., Pierce, M. B., Khan, A. R., Dirks, R. M., and Pierce, N. A. (2011) NUPACK: Analysis and design of nucleic acid systems. J. Comput. Chem. 32, 170-173.

(53) Talbot, E. L., Kotar, J., Parolini, L., Di Michele, L., and Cicuta, P. (2017) Thermophoretic migration of vesicles depends on mean temperature and head group chemistry. Nat. Comm. 8, 15351.

(54) Liu, A., and Qi, X. (2012) Molecular Dynamics Simulations of DOPC Lipid Bilayers: The Effect of Lennard-Jones Parameters of Hydrocarbon Chains. Computational molecular bioscience 2, 78-82.

(55) Whitelam, S., and Geissler, P. L. (2007) Avoiding unphysical kinetic traps in Monte Carlo simulations of strongly attractive particles. J. Chem. Phys. 127, 154101.

(56) Chodera, J. D. (2016) A Simple Method for Automated Equilibration Detection in Molecular Simulations. J. Chem. Theory Comput. 12, 1799-1805. 


\section{Graphical TOC Entry}

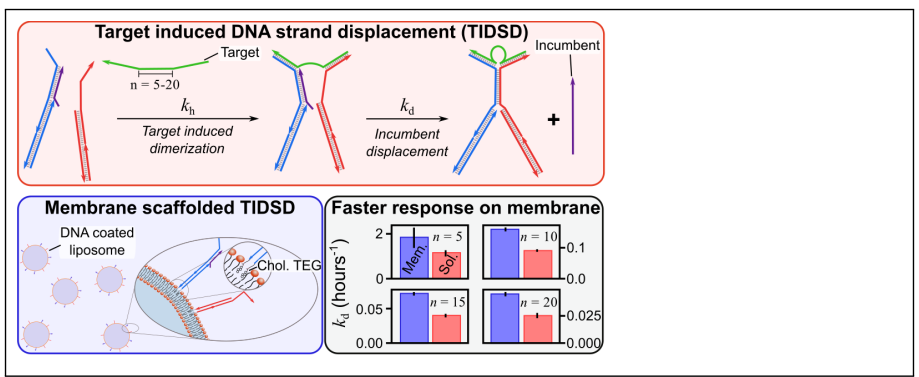

